Wagner de Jesus Martins

Elizabeth Artmann"

Francisco Javier Uribe Rivera"

\section{Gestão comunicativa para redes cooperativas de ciência, tecnologia e inovação em saúde}

\author{
Communication management of \\ collaborative networks of science, \\ technology and innovation in health
}

Fundação Oswaldo Cruz (Fiocruz). Brasília, DF, Brasil

" Departamento de Administração e Planejamento em Saúde. Escola Nacional de Saúde Pública Sergio Arouca. Fiocruz. Rio de Janeiro, RJ, Brasil

\author{
Correspondência | Correspondence: \\ Wagner de Jesus Martins \\ Fiocruz \\ Av. L3 Norte \\ Campus Universitário Darcy Ribeiro, Gleba A \\ 70910-900 Brasília, DF, Brasil \\ E-mail: wjesusmartins@gmail.com
}

\section{RESUMO}

O objetivo do artigo foi propor um modelo de gestão comunicativa de redes para o Sistema de Inovação em Saúde. Para isso discute-se o complexo produtivo da saúde em sua relação com o desenvolvimento e apresentam-se algumas sugestões para o formato mais operacional da proposta. Utilizaram-se teóricos da linguagem, especialmente Habermas, e exemplos de outros países. Enfoques comunicativos e de negociação de compromissos, que ajudam a criar formas de coordenação e consensos fundamentados na argumentação crítica, poderiam contribuir para a consolidação de redes democráticas.

DESCRITORES: Comunicação em Saúde. Setor de Assistência à Saúde, organização \& administração. Gestão de Ciência, Tecnologia e Inovação em Saúde. Políticas e Cooperação em Ciência, Tecnologia e Inovação.

\begin{abstract}
The objective of the article was to propose a model of communication management of networks for the Health Innovation System in Brazil. The health production complex and its relationship with the nation's development are addressed and some suggestions for operationalization of the proposed model are also presented. The discussion is based on Habermas' theory and similar cases from other countries. Communication strategies and approaches to commitment dialogue for concerted actions and consensus-building based on critical reasoning may help strengthen democratic networks.
\end{abstract}

DESCRIPTORS: Health Communication. Health Care Sector, organization \& administration. Health Sciences, Technology and Innovation Management. Policies and Cooperation in Science, Technology and Innovation. 


\section{INTRODUÇÃO}

A constituição dos novos arranjos institucionais em pesquisa, desenvolvimento e inovação buscam a maximização dos recursos disponíveis e a integração de competências e de conhecimentos entre os atores e as organizações. Essa integração contribui para a criação de confiança entre eles, suscita mais velocidade na geração e difusão dos conhecimentos e estimula a inovação. Competências, conhecimento e inovação são considerados de grande valor para ampliação da competitividade. ${ }^{a}$ Para Gadelha, ${ }^{9}$ a capacidade de inovação é determinada pelo potencial de transformação de conhecimentos em bens e serviços novos ou melhorados em sua qualidade e/ou processo produtivo.

A inovação tem sido tema recorrente na discussão sobre o desenvolvimento econômico do País e recentemente foi introduzido na agenda do Sistema Único de Saúde (SUS) na forma de Subsistema Nacional de Inovação em Saúde, ${ }^{9}$ parte do Sistema de Inovação. ${ }^{5}$ Este é constituído por empresas, instituições de ensino, agências de pesquisa e de financiamento, tanto públicas quanto privadas. Por meio delas o governo formula e executa políticas de incentivo à inovação.

Essas instituições não mantêm necessariamente relações hierárquicas entre si e pertencem ao espaço do público e da sociedade civil, do organizativo formal e informal. A diversificação dessas instituições representa um desafio para a gestão desse sistema. O modelo de gestão mais adequado ao sistema é o de gestão de redes, ancorado na busca da cooperação e no exercício da comunicação intensiva. $\mathrm{O}$ objetivo do presente artigo foi propor um modelo de gestão comunicativa de redes para o Sistema de Inovação em Saúde. Para isso discute-se o complexo produtivo da saúde em sua relação com o desenvolvimento e apresentam-se algumas sugestões para o formato mais operacional da proposta.

\section{COMPLEXO PRODUTIVO DA SAÚDE E O DESENVOLVIMENTO}

Tradicionalmente o sistema de inovação em saúde se confunde com o conceito, dos anos 1980, de complexo industrial ou simplesmente de indústria da saúde. ${ }^{6} \mathrm{O}$ complexo industrial deveria ser regulado pelo Estado para garantir aos consumidores acesso aos bens e serviços e assim melhorar sua qualidade de vida.

Ao situar a questão da saúde no contexto do desenvolvimento nacional e da política industrial, Gadelha ${ }^{10}$ privilegia como espaço de análise o relacionamento sistêmico entre o setor industrial (de base química e biotecnológica e de base mecânica, eletrônica e de materiais) e o setor dos prestadores de serviços. O setor industrial (complexo industrial da saúde) foi enfocado por ser considerado um núcleo economicamente vulnerável da área. Para esse autor, ${ }^{10}$ ampliar a base científica e tecnológica do complexo industrial da saúde para atender às necessidades do sistema de saúde depende da constituição de uma base endógena de inovação em saúde e de uma indústria competitiva.

Mas, ao privilegiar o modelo de ampliação competitiva, com foco no desenvolvimento econômico, é preciso não descuidar do objetivo de desenvolvimento social. Para Vianna \& Elias, ${ }^{29}$ o desenvolvimento deveria incorporar as dimensões econômica e social de maneira integrada. Ainda, a ação sobre o processo de produção de tecnologias e produtos de saúde deveria incluir a garantia do acesso, pois, com a formação do complexo industrial da saúde, o setor se configura em um espaço de acumulação de capital. A forte interdependência econômica, política e institucional que se estabelece entre as indústrias e os serviços de saúde justifica a constituição de um sistema nacional de ciência, tecnologia e inovação em saúde, com um olhar diferenciado, em que se articule a lógica econômica com a lógica sanitária. ${ }^{29}$

A legislação brasileira fornece os subsídios legais para definir na estrutura do sistema nacional de saúde ações governamentais para incrementar o desenvolvimento científico e tecnológico por meio da promoção e do incentivo à pesquisa e à capacitação tecnológica. Cabe ao MS atuar diretamente no fomento da capacidade produtiva do setor, o que implica a formação de redes entre os órgãos decisórios, normativos e regulatórios e os centros de produção, investigação científica e desenvolvimento tecnológico. Tais ações têm por objetivo implementar e aperfeiçoar os processos de absorção de conhecimento científico pelas indústrias, pelos serviços de saúde e pela sociedade.

Nesse contexto, o conceito de Complexo Produtivo da Saúde (CPS) mostra-se apropriado por incorporar uma perspectiva abrangente para designar essa ampla articulação de setores voltados a fornecer insumos para a produção de saúde. Insere-se nele o setor industrial, identificado como o componente crítico dessa cadeia ${ }^{10} \mathrm{e}$ que possui dinâmica diferenciada, pois pode alavancar processos de geração de emprego, renda e contribuir para o desenvolvimento científico a partir das demandas do Estado e dos serviços de saúde.

O CPS pode ser delimitado como um complexo político institucional e econômico. Ele envolve atividades realizadas por organizações que mantêm relações intersetoriais de compra e venda de bens e serviços,

a Valle MG, Salles Filho S. Redes de inovação tecnológica: aportes da economia evolucionista e da nova economia institucional [citado 2010 jan 17]. Disponível em: http://www.fearp.usp.br/egna/resumos/Valle.pdf 
de geração e transferência de conhecimentos e de tecnologias. Insere-se num contexto político e institucional bastante particular dado pelas especificidades da área da saúde, que deve priorizar o atendimento das necessidades da população em detrimento da lógica empresarial de lucro. ${ }^{10}$

O CPS opera num contexto de globalização excludente e assimétrica, em que a dependência econômica aparece em diversas formas, inclusive na produção de bens e serviços em saúde. ${ }^{16}$ Assim, a indústria é um foco importante da estratégia para a superação da dependência de insumos externos e a redução da vulnerabilidade do SUS. ${ }^{28}$

Grandes esforços foram feitos para a incorporação da concepção de CPS na agenda do SUS articulando as políticas científicas e tecnológicas às políticas industriais e de inovação. Entretanto, apesar desses esforços, ainda não se consolidou uma associação virtuosa entre saúde e desenvolvimento. ${ }^{10}$ Isso significa que ainda não foi possível ao Brasil a consolidação do sistema nacional de inovação na saúde. .,30 $^{4}$

O Ministério da Saúde incluiu o desenvolvimento científico, tecnológico e de inovação em saúde como uma das estratégias da gestão desde 2003, quando fez a revisão das estratégias para a consolidação do SUS. Criou a Secretaria de Ciência, Tecnologia e Insumos Estratégicos (SCTIE), para realizar essa política, dentro de uma perspectiva sistêmica de longo prazo. Entre os objetivos da SCTIE estão redução da vulnerabilidade da política social brasileira, fortalecimento do CPS e aumento da competitividade em inovação dos produtores de insumos estratégicos para saúde.

Em 2004, na $2^{\mathrm{a}}$ Conferência Nacional de Ciência, Tecnologia e Inovação em Saúde foram aprovadas a Política Nacional de Ciência, Tecnologia e Inovação em Saúde e a Agenda Nacional de Prioridades de Pesquisa em Saúde, que deveria ser atualizada periodicamente para orientar a produção científica em saúde. Esse processo visou ampliar a indução de pesquisas, voltadas para as necessidades e problemas prioritários na agenda do SUS. ${ }^{b}$

Em 2006, a SCTIE passou a atuar na indução da produção nacional de insumos estratégicos e na regulação do segmento, intensificando a articulação da política de saúde com a política de desenvolvimento industrial.

Em 2008 foi criado o Grupo Executivo do Complexo Industrial da Saúde (GCIS) para ser o espaço de governança intersetorial da produção e inovação e estimular a constituição de relacionamentos e redes entre as diferentes unidades de produção de insumos e de conhecimento tecnológico (públicas e privadas).

Essas redes envolvem os segmentos governamental, não governamental e empresarial por meio de parceria público-privada, conforme o modelo de tripla hélice. ${ }^{c}$ Tal modelo objetiva a transferência da tecnologia para o mercado, tendo como pano de fundo o interesse público. Ademais, a estratégia do Ministério da Saúde assume um papel sistêmico e estrutural ao intervir sobre as relações de interdependências entre as organizações do complexo da saúde em favor da inovação e do desenvolvimento econômico. As formas de integração entre a indústria e serviços são fundamentais do ponto de vista das políticas de saúde para a incorporação da inovação desde o início do processo. ${ }^{30}$

É fundamental conjugar os sistemas nacionais de inovação com os sistemas subnacionais e os internacionais. Nessa medida, as redes de inovação apresentariam limites difusos e em permanente evolução. Os componentes subnacional e transnacional teriam uma participação decisiva na impulsão do processo de inovação, em que pese a importância permanente do fator nacional. ${ }^{15}$

\section{REDES COOPERATIVAS E A INTEGRAÇÃO COMUNICATIVA}

Um arranjo institucional que atue como apoio à gestão estratégica de redes cooperativas no contexto da saúde e que contribua para consolidar um sistema de ciência, tecnologia e inovação em saúde deve considerar as quatro características das mudanças organizacionais em curso: ${ }^{17} 1$ ) integração organizacional com configurações descentralizadas, mais achatadas e interconectadas em rede; 2) capacidade de realizar melhorias contínuas e a participação das pessoas e sua permanente capacitação para enfrentar e resolver os problemas; 3 ) agilidade e adaptabilidade nos processos produtivos, para responder às necessidades da demanda segmentada; 4) as organizações como sistemas abertos, criando vínculos sistêmicos de cooperação, redes interorganizacionais.

As organizações tornam-se redes, unidades e atores interconectados cada vez mais dependentes do estabelecimento de uma relação comunicacional entre usuários, fornecedores e concorrentes, i.e., uma rede de informação/comunicação para realização de ações pactuadas. Uma estrutura em rede implica um conjunto

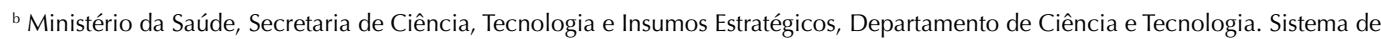
Informação de Ciência e Tecnologia em Saúde - versão 2.0.7. Brasília, DF: 2012. [citado 2012 out 18] Disponível em: http://bvsbms.gov.br/ bvs/publicações/AGENDA_PORTUGUESMONTADO.PDF

${ }^{c}$ Dal Poz MES. Da dupla a tripla hélice: o projeto Genoma Xylella [dissertação de mestrado]. Campinas: Instituto de Geociências da UNICAMP; 2000.
} 
de relações estáveis, de natureza não hierárquica e interdependente, vinculando atores com interesses comuns e que intercambiam recursos para concretizar esses interesses, com base na cooperação. ${ }^{7}$

Um dos grandes desafios do Ministério da Saúde na gestão do sistema de inovação é coordenar os produtores nacionais e criar formas de comunicação interorganizacional entre os participantes para orientar as decisões estratégicas. Coordenar refere-se ao processo de gerenciar dependências entre atividades. ${ }^{18}$

Com base em Habermas, ${ }^{12}$ a coordenação da ação dos participantes das redes de interação ancora-se na comunicação, envolvendo o agir comunicativo compreendido como um processo de entendimento a partir da linguagem, em que os atos de fala correspondem a pretensões de validade que podem ser aceitas ou não pelos participantes. O agir comunicativo articula duas formas: a comunicação pura - que implica o resgate automático dessas pretensões em função de um consenso acrítico garantido pelo pertencimento a um mesmo mundo da vida ${ }^{12}$ - e o discurso - que subentende a busca do consenso por meio da argumentação e exposição de razões. ${ }^{1,24,25}$

A compreensão linguística das organizações ${ }^{24,25}$ traz questões importantes para a gestão de redes. Para Flores, ${ }^{8}$ a organização é uma rede de conversações na qual predominam determinados atos de fala como as petições e os compromissos. Dois conceitos são fundamentais em sua construção: o de escuta, relacionado à dimensão cultural e à tradição, e o de rompimento referente a uma interrupção do fluxo normal da ação, ou das conversações para a ação. Por meio deles, é possível compreender o contexto organizacional e a antecipação de problemas. O Coordenador, criado por Flores, ${ }^{8}$ é um software que opera como administrador de conversações. Apoiado na taxonomia dos atos de fala de Austin $^{2}$ e Searle ${ }^{27}$ Flores $^{8}$ propõe alguns poucos módulos que operariam em pares (como oferta de ação/contraoferta, petição/promessa e outros). Isso facilita a elaboração de um aplicativo para pactuar compromissos de ação e monitorar permanentemente as conversações abertas. Esse instrumento é um excelente sistema de prestação de contas, pois informa de maneira atualizada em qual conversação existe uma promessa não cumprida, ou uma petição que não se traduziu em uma declaração de aceitação ou numa contraproposta, por exemplo.

Lima $^{18}$ analisa a coordenação de serviços de saúde por meio de um modelo construído a partir da lógica do agir comunicativo e baseado na inter-relação entre interdependência, coordenação e integração. Seu eixo estruturante é uma rede dinâmica de conversações que se estabelece entre os distintos atores do sistema. A ideia de rede de conversações como operadora da atividade de coordenar interdependências e promover integração fornece uma oportunidade de análise a partir das conversações que se estabelecem nas distintas dimensões de integração do sistema. $\mathrm{O}$ autor rastreia as redes de conversações que se estabelecem a partir de determinados rompimentos (quiebres) ${ }^{9}$ na experiência analisada de um serviço de atendimento móvel de urgência regional, identificando os atos de fala predominantes nessas redes: juízos, afirmações, declarações, ofertas, promessas e os principais grupos de atores envolvidos nos fluxos de conversação catalogados, assim como as possibilidades de encaminhamento positivo dos rompimentos em termos de compromissos.

A aplicação dessa proposta para a coordenação das redes que constituem o Sistema Nacional de Ciência e Tecnologia em Saúde (SNCTIS) permitiria construir e acompanhar uma agenda de prioridades ancorada na escuta e interpretação das demandas sociais a partir dos atores e instituições envolvidas.

\section{MODELO DE GESTÃO PARA AS REDES DE CT\&I EM SAÚDE}

Morel $^{20}$ destaca a importância de se discutirem o perfil e as características básicas do SNCTIS, quais sejam: 1) articulação dos principais atores e instituições atuantes nos campos da ciência e tecnologia (C\&T) e da saúde em redes; 2) estrutura organizacional do sistema; e 3) sua governança. A seguir discutem-se a questão do modelo de coordenação e a estrutura organizacional que deveria assumir o SNCTIS.

O envolvimento de comunidades de linguagem tão diferente no processo de gestão das tecnologias sugere a necessidade de um modelo de apoio à gestão que permita um fluxo contínuo de comunicações/informações. Além disso, deve orientar as ações desses atores e promover a colaboração entre o poder político e os órgãos da sociedade civil.

Atualmente, a criação de mecanismos para permitir maior colaboração entre governo e as organizações empresariais e da sociedade civil encontra um cenário favorável. Porém a persistência de um Estado ainda permeável aos interesses dos mais poderosos tende a dificultar que a coordenação das redes seja realizada por órgãos do executivo com poder normatizador. Estes, usualmente, encontram dificuldades para mediar interesses tão díspares e constituir de forma comunicativa uma agenda política, o que abre espaço para que setores privados tentem impor seus interesses.

Surge, assim, a necessidade de uma institucionalidade que tenha um relativo insulamento referente aos interesses privados em interação. Algumas características são desejáveis, como: elevada estabilidade política e técnica e, ao mesmo tempo, flexibilidade e permeabilidade às mudanças; confiabilidade perante os atores envolvidos na rede; reconhecida capacidade 
técnico-científica no setor saúde e facilidade de interlocução com amplo espectro de atores dos diferentes setores da saúde; capacidade de moderação, integração, dinamização e catalisação. Entretanto, um insulamento extremado, como, por exemplo, a criação de órgão gestor ou regulador, não seria o mais adequado para a constituição de uma instância que integre, dinamize e catalise os diferentes processos realizados nas redes cooperativas. Essa instância ou agência seria denominada como de integração estratégica.

Na mesma linha, Fleury \& Ouverney ${ }^{7}$ apontam que o governo central deve atuar apenas como mediador e facilitador nas redes de políticas com governança descentralizada. Assim, uma agência que não se sobreponha sobre os demais membros pode ser designada para atuar na coordenação das interdependências.

O apoio à integração estratégica seria suportado por um sistema de informação e comunicação no ciberespaço, com base num modelo de gestão comunicativa. ${ }^{24}$ Isso facilitaria a formulação de estratégias, a tomada de decisão pactuada, o acompanhamento sistemático de projetos e ações, a integração de conhecimento e de competências para gerar inovações e a conversação entre os participantes.

Esse sistema de comunicação e de informação deveria estar aberto à noção de espaço público no sentido habermasiano, reunindo integrantes da sociedade civil para formação de opinião pública. Ancorado numa estrutura comunicacional do agir voltado para o entendimento, seria capaz de influenciar o poder político. ${ }^{22} \mathrm{O}$ espaço público constitui-se em uma estrutura discursiva de mediação entre a política e a sociedade que integra espaços coletivos e todas as mídias, incluindo as digitais, que seriam formas generalizadas de comunicação.

O segundo aspecto importante do modelo de gestão refere-se à utilização de ferramentas estratégicas de análises de redes para melhorar sua capacidade de organização e de elaboração de políticas. Morel et $\mathrm{al}^{21}$ aplicam a análise de redes sociais (baseada nos softwares NetDraw e Pajek) para caracterização das relações de coautoria entre instituições de pesquisa e apresentam como exemplos a Rede de Pesquisadores de Dengue, 2001-2008 e a Rede de Instituições de Tuberculose, 2006-2007 (Figura).

Na Espanha, o Instituto de Saúde Carlos III (principal órgão público de pesquisa em saúde), por meio da sua Subdireção Geral de Redes e Centros de Investigação Cooperativa, instituiu o programa das Redes Temáticas de Pesquisa Cooperativa em Saúde (Retics) ${ }^{26}$ em 2007. As Retics são compostas por grupos de pesquisas de diferentes esferas de governos e instituições de C\&T para atuar de forma colaborativa em torno dos temas prioritários da agenda de pesquisa.

Dentro dessa perspectiva, destaca-se a metodologia de análise de redes de conversações, ${ }^{18}$ que utiliza os conceitos de cliques e centralidade, por serem importantes para localizar o papel dos atores no processo de coordenação de ações no interior do sistema. Essa metodologia ajudaria a identificar os fluxos de conversações abertas, os atores envolvidos, os problemas discutidos e os objetivos e compromissos negociados.

O terceiro elemento do modelo corresponde à necessidade de investir no desenvolvimento de aplicativos para reforçar o sistema de informação e de comunicação no ciberespaço (modelo de webgestão). A lógica deles seria a de ajudar a promover a coordenação, incentivando o desenvolvimento de compromissos coletivos e a prestação de contas. A racionalidade do software Coordenador ${ }^{8}$ como instrumento de administração de conversações poderia ser útil. A Fiocruz está implementando uma adaptação desse software, baseada na proposta de Príncipe. ${ }^{\mathrm{d}}$ Estudos avaliativos do grau de coordenação de programas integrados de pesquisa e desenvolvimento tecnológico de instituições públicas acusam uma carência significativa dessas ferramentas, ${ }^{28}$ sendo crucial pesquisar e propor ferramentas atualizadas em formatos interativos de comunicação e gestão da informação.

Esse modelo de gestão deveria aproveitar ferramentas de gestão estratégica que operam como práticas a serviço da problematização de situações e da reflexão estratégica, incluindo a prospectiva estratégica, a análise estratégica hospitalar e o planejamento situacional. ${ }^{25}$

Vários autores ${ }^{3,19}$ destacam que a análise prospectiva constitui-se num instrumento importante a inovação e desenvolvimento. São várias iniciativas nacionais de planejamento de médio e longo prazo de $\mathrm{C} \& \mathrm{~T}$, como os estudos prospectivos da França, Alemanha, Reino Unido, Japão e Austrália, entre outros. Esses autores se referem ao conjunto de ferramentas de prospecção (Tecnological Foresight) como um modelo de colaboração e comunicação de pesquisadores voltados para a construção de uma visão de futuro.

\section{CONSIDERAÇÕES FINAIS}

Apesar de algumas redes mais sistematizadas, ${ }^{21} \mathrm{O}$ Brasil ainda não dispõe de um sistema nacional de inovação em saúde na sua plenitude, como já apontado. Vários autores ${ }^{11,14,23}$ sustentam que a produção científico-tecnológica em saúde depende, em grande

d Príncipe AS. A gestão da informação para apoio à decisão no setor público de saúde: uma proposta de sistema informatizado para a comunicação de compromissos institucionais na Fiocruz [dissertação de mestrado]. Rio de Janeiro: Escola Nacional de Saúde Pública Sérgio Arouca; 2005. 
Rede de pesquisadores de dengue, 2001-2008

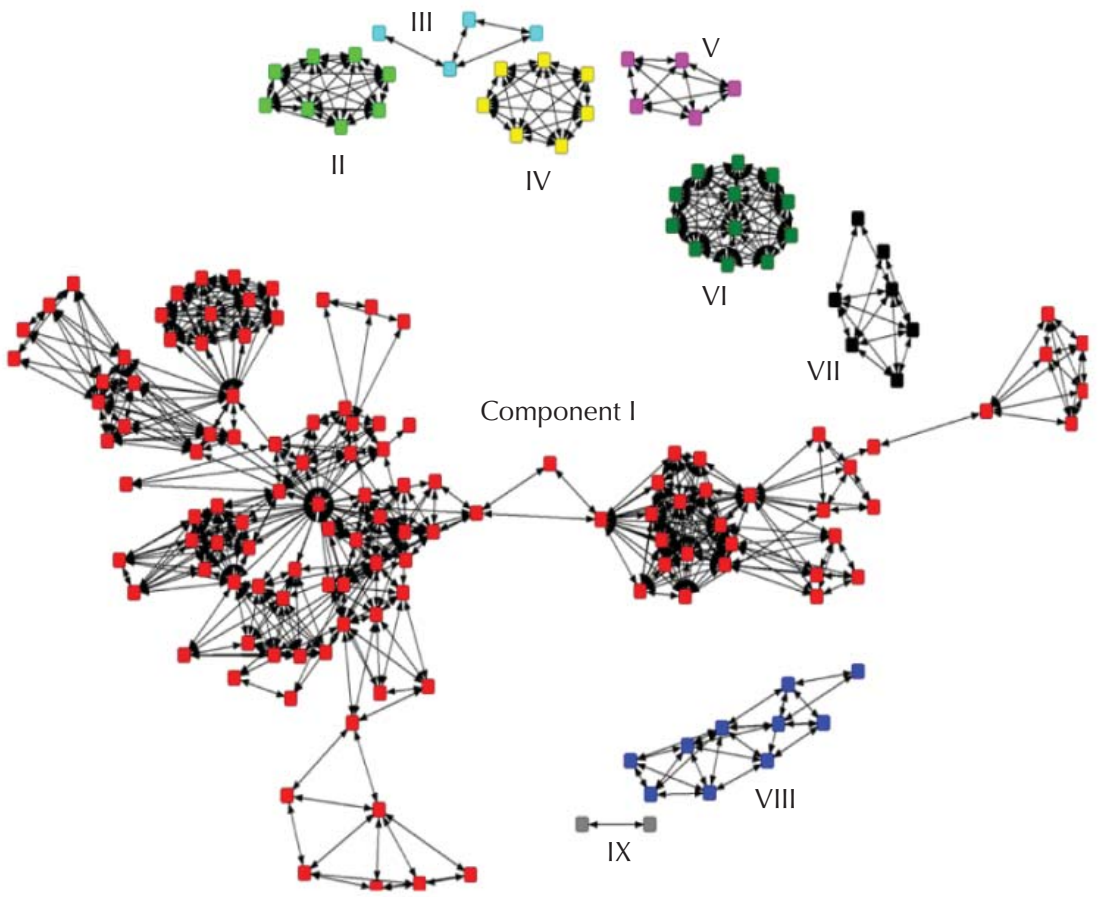

Rede de instituições de tuberculose, 2006-2007

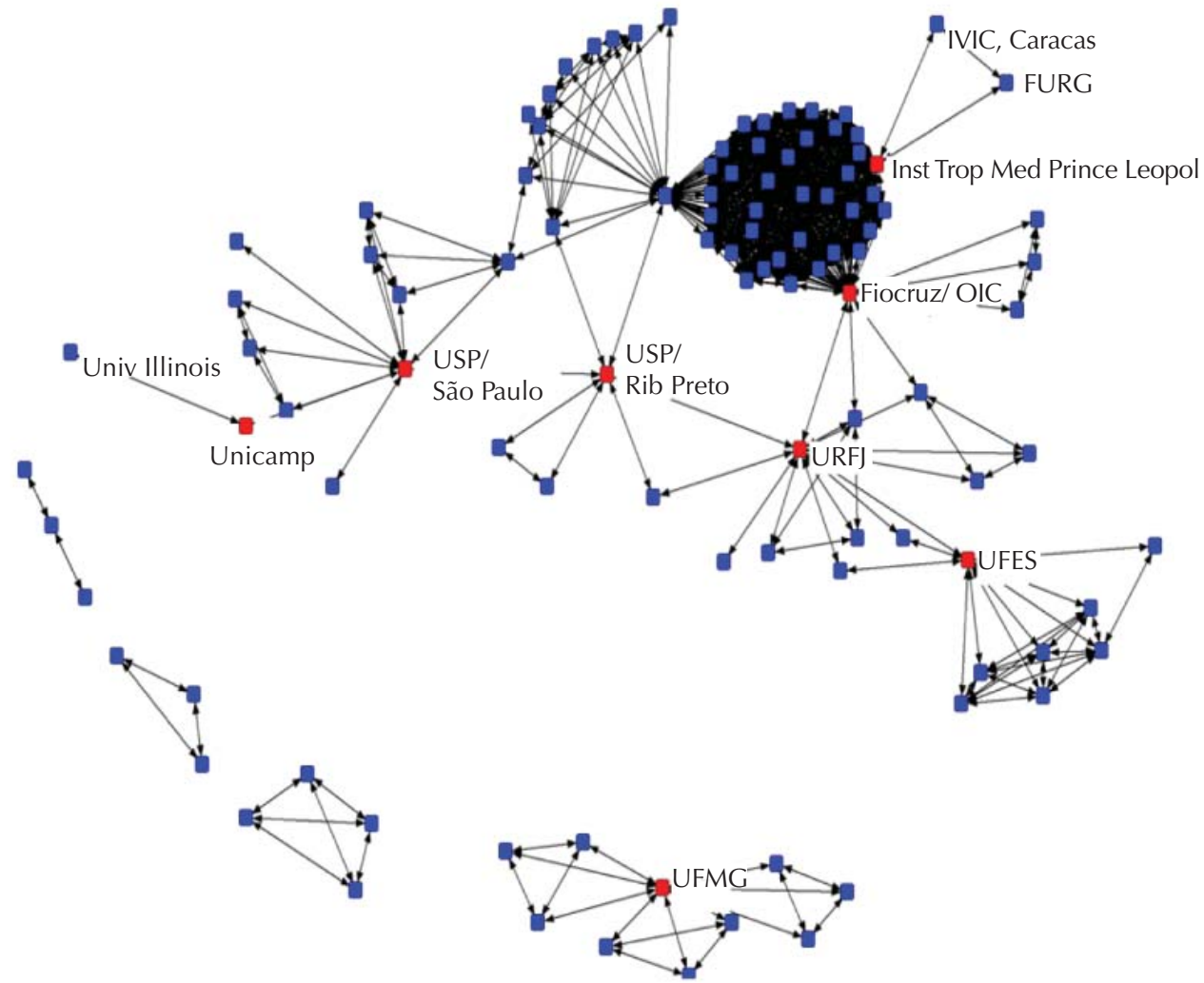

Figuras extraídas de Morel et al ${ }^{21}$ (2009)

Figura. Relações de co-autoria entre instituições de pesquisa de dengue e tuberculose. 
medida, do estabelecimento efetivo de redes, envolvendo uma interação complexa entre universidades, indústria e sistemas de assistência médica. Hicks \& Katz, ${ }^{13}$ especificamente, referem-se a um sistema biomédico de inovação, destacando a importância dos hospitais britânicos na produção científica da área, com duas redes superpostas que impulsionam a inovação em saúde: de um lado, os hospitais, as autoridades sanitárias regionais e a Pesquisa Médica de Pós-Graduação (responsável por 25\% da produção na década de 1980) e, de outro lado, a rede que articula o governo, as universidades e as indústrias. Esses trabalhos também destacam a importância da participação das empresas nacionais e transnacionais no processo de produção científica e tecnológica do setor, o que marcaria uma forte diferença com o que ocorre no Brasil, que tem uma indústria específica pouco competitiva. ${ }^{10}$ Para Nelson ${ }^{23}$ há necessidade de um forte entrelaçamento entre ciência e tecnologia para a pesquisa e inovação médica, a exemplo da biotecnologia. Por outro lado, esse autor assinala que a inovação médica é crescentemente dependente de pesquisas interdisciplinares, como o caso dos medicamentos e o dos equipamentos médicos. No Brasil, a ainda baixa participação relativa dos hospitais e indústrias no processo de inovação, aliada ao pouco conhecimento que se tem dessa participação, mostra uma associação pouco virtuosa entre saúde e desenvolvimento. Esse fato e os obstáculos à constituição plena de redes, embora em crescimento,

\section{REFERÊNCIAS}

1. Artmann E. Interdisciplinaridade no enfoque intersubjetivo habermasiano: reflexões sobre planejamento e AIDS. Cien Saude Coletiva. 2001;6(1):183-95. DOI:10.1590/S1413-81232001000100015

2. Austin JL. How to do things with words. Oxford: Oxford University Press; 1962.

3. Canongia C, Pereira MNF, Antunes A. Uso e gestão da informação na prospecção em medicamentos contra câncer e mama. Parcerias Estrateg. 2002;7(15):93-109.

4. Cassiolato JE, Lastres HMM, Maciel ML. Systems of innovation and development: evidence from Brazil. Cheltenham: Edward Elgar; 2003.

5. Cassiolato JE, Lastres HMM. Sistemas de inovação e desenvolvimento: as implicações de política. São Paulo Perspect. 2005;19(1):34-45. DOI:10.1590/S0102-88392005000100003

6. Cordeiro H. A indústria de saúde no Brasil. Rio de Janeiro: Graal; 1980.

7. Fleury S, Ouverney AM. Gestão de redes: a estratégia de regionalização da política de saúde. Rio de Janeiro: Editora FGV; 2007.

8. Flores F. Inventando la empresa del siglo XXI. 5.ed. Santiago, Chile: Hachette; 1993.

9. Gadelha CAG, Quental C, Fialho BC. Saúde e explicariam a dificuldade de se conformar o Sistema Nacional de Inovação em Saúde. Nos sistemas de inovação em saúde da Europa ${ }^{15} \mathrm{e} \mathrm{de} \mathrm{Cuba}{ }^{14}$ há uma forte ênfase na consolidação de polos científico-produtivos, como condição de possibilidade de uma perspectiva sistêmica de estímulo à inovação.

O Estado teria um papel fundamental na regulação e coordenação do sistema de inovação. A necessidade desse papel proeminente está relacionada ao seu caráter de grande consumidor de produtos de inovação; à sua capacidade de veicular demandas de inovações segundo necessidades sociais; à sua capacidade de agilizar a comunicação e circulação de informação entre os componentes do sistema; e com a sua atuação econômica na linha de um planejamento indicativo, ${ }^{25}$ que interfere positiva ou negativamente na propensão a consumir e investir da indústria.

Esse papel de regulação e coordenação se aproxima mais de um trabalho de mediação, sem excesso de normatividade e burocracia.

A diversidade de instituições que compõem as redes no contexto de inovação em saúde exige a utilização de enfoques comunicativos e de negociação de compromissos. Somente estes poderiam criar formas de coordenação e consensos fundamentados na argumentação crítica e contribuir para a consolidação de redes democráticas capazes de responder aos interesses da população.

inovação: uma abordagem sistêmica das indústrias de saúde. Cad Saude Publica. 2003;19(1):47-59. DOI:10.1590/S0102-311X2003000100006

10. Gadelha CAG. Desenvolvimento, complexo industrial da saúde e política industrial. Rev Saude Publica. 2006;40(Esp):11-23. DOI:10.1590/S0034-89102006000400003

11. Gelijns A, Rosenberg N. The changing nature of medical technology development. In: Gelijns A, Rosenberg N, editors. Sources of medical technology: universities and industry. Washington, DC: National Academy Press: 1995. p.3-14. (Medical Innovation at the Crossroads, 5).

12. Habermas J. Teoria de la acción comunicativa. Madrid: Taurus; 1987.

13. Hicks D, Katz J. Hospitals: the hidden research system. Sci Public Policy. 1996;23(5):297-304.

14. Jover J, Sánchez FC, Ones IP, Arriete LFM. Ciencia, tecnología y sociedad em Cuba: construyendo una alternativa desde la propiedad social. In: Gallina A, Jover JN, Capecchi V, Arriete LFM. Innovaciones creativas y desarrollo humano. Montevideo: Ediciones Trilce; 2006. p.185-209.

15. Kuhlman S. Future governance of innovation policy in Europe: three scenarios. Res Policy. 2001;30(6):95376. DOI:10.1016/S0048-7333(00)00167-0 
16. Lastres HMM, Ferraz JC. Economia da informação, do conhecimento e do aprendizado. In: Lastres H, Albagli S, coordenadores. Informação e globalização na era do conhecimento. Rio de Janeiro: Campus; 1999. p.27-57.

17. Perez C. Cambio técnico, restructuración competitiva y reforma institucional en los países en desarrollo. Trimestre Econ. 1992;59(233):23-64.

18. Lima JC, Rivera FJU. Redes de conversação e coordenação de ações de saúde: estudo em um serviço móvel regional de atenção às urgências. Cad Saude Publica. 2010;26(2):323-36. DOI:10.1590/S0102-311X2010000200011

19. Martin BR, Johnston R. Technology foresight for wiring up the National Innovation System: experiences in Britain, Australia, and New Zealand. Technol Forecast Soc Change. 1999;60(1):37-54. DOI:10.1016/S0040-1625(98)00022-5

20. Morel CM. A pesquisa em saúde e os objetivos do milênio: desafios e oportunidades globais, soluções e políticas nacionais. Cienc Saude Coletiva. 2004;9(2):261-70. DOI:10.1590/S1413-81232004000200002.

21. Morel CM, Serruya SJ, Penna GO, Guimarães R. Co-authorship network analysis: a powerful tool for strategic planning of research, development and capacity building programs on neglected diseases. PLoS Neg/ Trop Dis. 2009;3(8):e501. DOI:10.1371/journal.pntd.0000501

22. Müller Neto JS, Artmann E. Política, gestão e participação em saúde: reflexão ancorada na teoria da ação comunicativa de Habermas. Cienc Saude Coletiva. 2012;17(12):3689-98.

23. Nelson R. The intertwining of public and proprietary in medical technology. In: Gelijns A, Rosenberg N, editors. Sources of medical technology: universities and industry. Washington, DC: National Academy Press; 1995. p.219-p.220 (Medical Innovation at the Crossroads, 5).

24. Rivera FJU. Análise estratégica em saúde e gestão pela escuta. Rio de Janeiro: Editora Fiocruz; 2006, 2.ed.

25. Rivera FJU, Artmann E. Planejamento e gestão em saúde: histórico e tendências com base numa visão comunicativa. Cienc Saude Coletiva. 2010;15(5):226574. DOI:10.1590/S1413-81232010000500002

26. Sánchez PL, Fernández-Baza A, Fernández-Avilés F. ¿Qué son las redes temáticas de investigación cooperativa en salud (RETICS)? Cardiocore. 2011;46(4):131-5.

27. Searle JR. A taxonomy of illocutionary acts. In: Gunderson K, editor. Language, mind and knowledge. Minneapolis: University of Minnesota Press; 1975. p.344368 (Minnesota Studies in the Philosophy of Science, 7).

28. Teixeira MO, Machado CJS, Filipecki ATP, Cortes BA, Klein HE. Descrição e análise do uso de um instrumento de coordenação em um instituto público de pesquisa em biomedicina. Cienc Saude Coletiva. 2011;16(3):1835-47. DOI:10.1590/S1413-81232011000300019

29. Viana ALD, Elias PEM. Saúde e desenvolvimento. Cienc Saude Coletiva. 2007;12(Supl):1765-77. DOI:10.1590/S1413-81232007000700002

30. Viana ALD, lozzi FL, Albuquerque MV, Bousquat A. Saúde, desenvolvimento e inovação tecnológica: nova perspectiva de abordagem e de investigação. Lua Nova. 2011;(83):41-77. DOI:10.1590/S0102-64452011000200003

Artigo submetido ao processo de julgamento por pares adotado para qualquer outro manuscrito submetido a este periódico, com anonimato garantido entre autores e revisores. Editores e revisores declaram não haver conflito de interesses que pudesse afetar o processo de julgamento do artigo.

Os autores declaram não haver conflito de interesses. 